\title{
Coherent axion-photon transformations in the forward scattering on atoms
}

\author{
V. V. Flambaum, ${ }^{1,2,3}$ I. B. Samsonov, ${ }^{1,4}$ H. B. Tran Tan, ${ }^{1}$ and D. Budker ${ }^{2}$ \\ ${ }^{1}$ School of Physics, University of New South Wales, Sydney 2052, Australia \\ ${ }^{2}$ Johannes Gutenberg-Universität Mainz, 55099 Mainz, Germany \\ ${ }^{3}$ The New Zealand Institute for Advanced Study, Massey University Auckland, \\ 0632 Auckland, New Zealand \\ ${ }^{4}$ Bogoliubov Laboratory of Theoretical Physics, JINR, Dubna, Moscow region 141980, Russia
}

(Received 23 August 2018; published 27 November 2018)

\begin{abstract}
In certain laboratory experiments the production and/or detection of axions is due to the photon-axion transformations in a strong magnetic field. This process is coherent, and the rate of the transformation is proportional to the length $l$ and magnitude $B$ of the magnetic field squared, $\sim l^{2} B^{2}$. In the present paper, we consider coherent production of axions due to the forward scattering of photons on atoms or molecules. This process may be represented as being due to an effective electromagnetic field which converts photons to axions. We present analytical expressions for such effective magnetic and electric fields induced by resonant atomic M0 and M1 transitions, as well as give some numerical estimates for these fields. The corresponding experiments would allow one to measure the electron-axion coupling constant $g_{a e}$ in the same way as the photon-axion coupling $g_{a \gamma}$ is studied.
\end{abstract}

DOI: 10.1103/PhysRevD.98.095028

\section{INTRODUCTION}

The axion was introduced to explain the absence of $C P$ violation in the strong interaction [1-9] and is considered as a probable candidate for a dark matter particle. The majority of experiments searching for axions in laboratories are based on the transformation of axions to photons (for axion detection) and photons to axions (for axion production) in a strong magnetic field [10-49]. The use of atomic and molecular transitions for the axion detection has been suggested in Refs. [50-55]. It is natural to assume that the atomic transitions can play role of effective electromagneic field in the process of conversion of photons to axions. This paper aims to estimate such effective electric and magnetic fields induced by forward scattering of photons on atoms near atomic resonances.

The photon-to-axion conversion probability in magnetic field $B$ is [56] (in units $\hbar=c=1$ )

$$
P=\frac{\omega}{4 k_{a}}\left(g_{a \gamma} B l\right)^{2} F^{2}(q),
$$

where $\omega$ is the axion and photon energy, $k_{a}$ is the axion momentum, $g_{a \gamma}$ is the axion-photon coupling constant, $l$ is the spatial extent of the magnetic field in the direction of the

Published by the American Physical Society under the terms of the Creative Commons Attribution 4.0 International license. Further distribution of this work must maintain attribution to the author(s) and the published article's title, journal citation, and DOI. Funded by SCOAP . axion-photon beam, $F(q)=\int d x e^{-i q x} B(x) / \bar{B} l$ is the form factor, $q=\omega-k_{a}$ is the momentum transferred from the photon field to the axion field. For a small axion mass, $m_{a} \ll \omega$, we have $q \approx 0$ and $F(0) \approx 1$.

Probabilities of the transformation of photons to axions and axions to photons are calculated by solving the system of Maxwell and Klein-Gordon equations coupled through the interaction term in the Lagrangian density:

$$
L^{a \gamma}=g_{a \gamma} a(\mathbf{E} \cdot \mathbf{B}),
$$

where $a, E$ and $B$ are the axion, electric and magnetic fields, correspondingly. A similar effective Lagrangian density appears due to the interaction between the axion field and an atom

$$
L_{\mathrm{eff}}^{a \gamma}=g_{a e} a\left(\mathbf{E} \cdot \mathbf{B}_{\mathrm{eff}}+\mathbf{E}_{\mathrm{eff}} \cdot \mathbf{B}\right),
$$

where the effective magnetic $\mathbf{B}_{\text {eff }}$ and electric $\mathbf{E}_{\text {eff }}$ fields are produced by the atom, while $\mathbf{E}$ and $\mathbf{B}$ are physical electromagnetic fields of an external photon. The effective Lagrangian (3) includes the coupling constant $g_{a e}$ which originates from the axion-electron (or axion-nucleon) interaction

$$
L^{a e}=g_{a e} \partial_{\mu} a \bar{\psi} \gamma^{5} \gamma^{\mu} \psi
$$

where $\psi$ is the electron (or nucleon) spinor field. The aim of this paper is to derive the expressions for the effective electric and magnetic fields stipulated by the interaction (4). 
The rest of the paper is organized as follows. In the next section we derive analytical expressions for the effective magnetic and electric fields due to the photon-axion transformation. The numerical estimates of these fields in vapor, liquid and solid media are given in Sec. III. The obtained results are summarized in Sec. IV. In the Appendices, we present the details of calculations of the effective fields.

\section{CAlCulations}

Allowing for the axion-electron interaction (4), one can consider a scattering amplitude, where a bound electron absorbs an incident photon and emits an axion. Of special interest is the scattering in forward direction since the forward scattering on multiple atoms is always coherent. If the wavelengths of the photon and axion are significantly larger than the distance between the atoms, we may treat the atomic system as a continuous medium, and the forward scattering amplitude scales linearly with the number density of atoms $N_{\text {at }}$. Thus, the effective Lagrangian (3) can be identified with the amplitude of forward scattering of photons on atoms [57]

$$
L_{\text {eff }}^{a \gamma}=N_{\text {at }} \sum_{n} \frac{M_{0 n}^{a} M_{n 0}^{\gamma}}{E_{0}-E_{n}+\omega-i \Gamma_{n} / 2},
$$

where the summation goes over the atomic (or nuclear) excited states $n$ with the energies $E_{n}$ and widths $\Gamma_{n}$. Here $\omega$ is the energy of the incident photon, $M_{0 n}^{a}$ is the matrix element of the axion-electron interaction (4) and $M_{n 0}^{\gamma}$ is the matrix element of the interaction of atomic electrons with external electromagnetic field of the incident photon.

The matrix element $M_{0 n}^{a}$ in Eq. (5) is given by $M_{0 n}^{a}=\left\langle 0\left|H^{a e}\right| n\right\rangle$, where the Hamiltonian $H^{a e}$ corresponds to the interaction (4). For non-relativistic electrons this Hamiltonian has the form $[51,52]$

$$
H^{a e}=g_{a e}\left(\boldsymbol{\nabla} a \cdot \boldsymbol{\sigma}-\partial_{t} a \frac{\mathbf{p} \cdot \boldsymbol{\sigma}}{m_{e}}\right),
$$

where $m_{e}$ is the electron mass and $\mathbf{p}$ is its momentum. The form of this Hamiltonian can be further specified when the axion field is given by the plane wave with energy $\omega$ and wave vector $\mathbf{k}, a(\mathbf{r}, t)=a_{0} \sin (\omega t-\mathbf{k r})$. Then the Hamiltonian may be represented in the form of multipole expansion with the leading terms given by (modulo the oscillating factor $e^{i \omega t}$ )

$$
\begin{gathered}
H^{a e}=g_{a e} a_{0}\left(H_{\mathrm{M} 0}^{a e}+H_{\mathrm{M} 1}^{a e}+\ldots\right), \\
H_{\mathrm{M} 0}^{a e}=\frac{1}{2}\left(-\frac{\omega}{m_{e}} \mathbf{p} \cdot \boldsymbol{\sigma}+i(\mathbf{k} \cdot \mathbf{r})(\mathbf{k} \cdot \boldsymbol{\sigma})\right), \\
H_{\mathrm{M} 1}^{a e}=-\frac{1}{2}(\mathbf{k} \cdot \boldsymbol{\sigma}) .
\end{gathered}
$$

The scattering amplitude (5) may be considered as a process, where an atom is (virtually) excited by absorbing a photon and returns back to the initial state via axion emission. Note that the M0 axions induce transitions between the states of opposite atomic parity but the same total angular momentum. Thus, M0 axions are produced when atoms absorb E1 photon which also changes the parity. Analogously, M1 axions do not change parity, and they may be produced when M1 photons are absorbed. As a result, in Eq. (5) the matrix element of the operator $H_{\mathrm{M} 0}^{a e}$ should be coupled with the matrix element of E1 photon absorption operator $H_{\mathrm{E} 1}^{\gamma}=e(\mathbf{\epsilon} \cdot \mathbf{r})$, while the matrix element of the operator $H_{\mathrm{M} 1}^{a e}$ should be multiplied by the matrix element of M1 photon-absorption operator $H_{\mathrm{M} 1}^{\gamma}=\frac{e}{2 m_{e}}(\mathbf{n} \times \mathbf{\epsilon})(\mathbf{J}+\mathbf{S})$, where $\mathbf{n}$ is the propagation unit vector of the photon and $\boldsymbol{\epsilon}$ is its polarization. The former case corresponds to the effective magnetic field while the latter generates the effective electric field due to the interaction of photons with atoms,

$$
\begin{aligned}
& B_{\text {eff }}=N_{\mathrm{at}} \operatorname{Re} \sum_{n} \frac{\left\langle 0\left|H_{\mathrm{M} 0}^{a e}\right| n\right\rangle\left\langle n\left|H_{\mathrm{E} 1}^{\gamma}\right| 0\right\rangle}{E_{0}-E_{n}+\omega-i \Gamma_{n} / 2}, \\
& E_{\text {eff }}=N_{\mathrm{at}} \operatorname{Re} \sum_{n} \frac{\left\langle 0\left|H_{\mathrm{M} 1}^{a e}\right| n\right\rangle\left\langle n\left|H_{\mathrm{M} 1}^{\gamma}\right| 0\right\rangle}{E_{0}-E_{n}+\omega-i \Gamma_{n} / 2} .
\end{aligned}
$$

The direction of the effective fields is specified by the matrix elements in Eqs. (10) and (11). In fact, as we prove in our previous paper [55], for nonpolarized atoms the product of axion and photon matrix elements always sums to zero, $\sum_{n}\left\langle 0\left|H^{a e}\right| n\right\rangle\left\langle n\left|H^{\gamma}\right| 0\right\rangle=0$, because the excited states $|n\rangle$ with different projections of the total momentum contribute destructively. To get a nonvanishing result we have to assume that a (weak) external magnetic field $B_{2}$ is applied, which lifts the degeneracy of Zeeman sublevels such that only some of them are in resonance with the applied laser field. In this case, it is possible to show that the direction of the effective field $B_{\text {eff }}$ coincides with the direction of the applied physical magnetic field $B_{2}$ while $\mathbf{E}_{\text {eff }}$ is directed along $\mathbf{k} \times \mathbf{B}_{2}$.

A resonant atomic transition may lead to a significant photon absorption and a phase shift of the photon field (relative to the axions field) which destroys the coherence. Both effects may be seen from the complex phase in the photon field $A \propto e^{i n_{r} \mathbf{k} \cdot \mathbf{r}}$, where

$$
n_{r}=1-2 \pi N_{\text {at }} \sum_{n} \frac{\left|M_{n 0}^{\gamma}\right|^{2}}{E_{0}-E_{n}+\omega-i \Gamma_{n} / 2}
$$

is the complex refractive index. One could go to the tail of resonance to suppress the photon absorption since the imaginary part of $n_{r}$ decreases quadratically with $E_{0}-E_{n}+\omega$, but the phase decreases slowly (linearly), similar to $E_{\text {eff }}$ and $B_{\text {eff }}$ in Eqs. (10) and (11). The phase of 
the photons may be corrected by placing phase-correcting transparent plates in the medium. This situation is opposite to the case of photon-axion transformation in magnetic field [58], where it was proposed to use a gas to match the photons' speed with the speed of low-mass axion. In our case the phase-correcting transparent plates may be needed if the photons' phase velocity in the medium appears less than the axion's speed. In this case the plate should add the photons' phase to equate the phase difference with axions to a multiple of $2 \pi$.

\section{NUMERICAL ESTIMATES}

Note that the effective fields (10) and (11) are proportional to the atom density $N_{\text {at }}$. Thus, a stronger effective field is produced in dense atomic systems such as solids, liquids or dense gases. In this note we consider noble gases in liquid phase and heavy metal vapors since they can be treated using the standards atomic methods. We also show that there is a significant enhancements of the effective field in crystals with narrow spectral lines.

\section{A. Effective magnetic field in liquid xenon}

Let us consider liquid xenon with atomic density $N_{\text {at }}=1.3 \times 10^{22} \mathrm{~cm}^{-3}$. The resonant axion M0 transition is possible from the ground state $0^{+}$to the excited state $0^{-}$ with the energy $E_{0^{-}}=9.45 \mathrm{eV}$, while the corresponding photon transition is highly forbidden. This photon transition may be open when an external magnetic field $B_{2}$ is applied. This magnetic field causes the mixing of the states $0^{-}$and $1^{-}$, where the latter corresponds to the energy level $E_{1^{-}}=9.57 \mathrm{eV}$. As we demonstrate in Appendix A 1, the effective magnetic field may reach the value

$$
B_{\text {eff }} \approx 1.2 \times 10^{-2} \mathrm{~T} \text {. }
$$

We stress that, according to Eq. (3), the strength of interaction of this effective field with the axion field is specified by the coupling constant $g_{a e}$, which is independent from the axion-photon constant $g_{a \gamma}$. Thus, although this effective field is much weaker than the physical magnetic field $B=5.3 \mathrm{~T}$ applied at the ALPS experiment [59], it may be significant in the processes of axion production or detection if $g_{a e} \gg g_{a \gamma}$.

\section{B. Effective electric field in vapor thallium}

To estimate the effective electric field (11) we consider a vapor of $\mathrm{Tl}$ atoms at temperature $T=1200^{\circ} \mathrm{C}$ and density $N_{\text {at }}=6.6 \times 10^{17} \mathrm{~cm}^{-3}$. This system proved useful in the study of weak-interaction-induced optical activity of heavy-metal vapors [60]. Indeed, the transition between the ground state $A$ with $J=\frac{1}{2}$ and the nearest excited state $B$ with $J=\frac{3}{2}$ is of M1 type. The energy difference between these states $\omega=0.966 \mathrm{eV}$ corresponds to nearinfrared region. In Appendix A 2 we give an estimate of the effective electric field corresponding to such atomic transitions:

$$
E_{\mathrm{eff}} \approx 1.5 \times 10^{5} \frac{\mathrm{V}}{\mathrm{cm}} .
$$

This field is still not competitive with the physical magnetic field used at ALPS experiments.

\section{Effective electric field in a crystal}

According to Eqs. (10) and (11), the strong effective fields are achieved in media with high atom density $N_{\text {at }}$ and small width $\Gamma$. Therefore it is natural to consider photon-toaxion transformations in crystals with narrow optical lines. One of such crystals based on $\mathrm{EuCl}_{3} \cdot 6 \mathrm{H}_{2} \mathrm{O}$ compound has recently been studied in [61]. Remarkable spectral properties of this crystal are due to the $4 \mathrm{f}$ valence electron shell of the $\mathrm{Eu}^{3+}$ ion $[62,63]$. In particular, in [61] it was shown that the optical ${ }^{7} F_{0} \rightarrow{ }^{5} D_{0}$ transition in such isotopically purified crystal may be as narrow as $25 \mathrm{MHz}$. However, we cannot employ this transition for axion production since it corresponds to the induced electric dipole type and does not obey the axion-amplitude selection rules. Instead, we consider the transition ${ }^{7} F_{0} \rightarrow{ }^{5} D_{1}$ with the energy $\omega=$ $2.36 \mathrm{eV}$ in the $\mathrm{Eu}^{3+}$ ion, which is of M1 type [62] and is allowed for both the photon and axion amplitudes. Note that the states ${ }^{7} F_{0}$ and ${ }^{5} D_{1}$ refer to the intermediate coupling scheme, and each of them is given by a linear combination of the LS-states according to [63].

Although there is no explicit data about the width of the ${ }^{7} F_{0} \rightarrow{ }^{5} D_{1}$ transition in the $\mathrm{EuCl}_{3} \cdot 6 \mathrm{H}_{2} \mathrm{O}$ crystal, we assume that it may be as narrow as for the ${ }^{7} F_{0} \rightarrow{ }^{5} D_{0}$ transition, $\Gamma \sim 10^{-7} \mathrm{eV}$. Then, taking into account that the density of Eu atoms in this crystal is $N_{\text {at }} \approx 2.7 \times 10^{21} \mathrm{~cm}^{-3}$ [61], in Appendix A 3 we estimate the effective electric field due to the photon-axion transformation:

$$
E_{\mathrm{eff}} \approx 1.7 \times 10^{9} \frac{\mathrm{V}}{\mathrm{cm}} .
$$

Thus, the effective field in crystals (15) is much stronger than that in vapor media (14) due to higher atom density and smaller linewidth.

We point out that Eq. (15) gives a rough estimate of the effective field because we do not know the exact width of the line corresponding to the ${ }^{7} F_{0} \rightarrow{ }^{5} D_{1}$ transition in the $\mathrm{EuCl}_{3} \cdot 6 \mathrm{H}_{2} \mathrm{O}$ crystal. We hope that a more accurate estimate may be given when this transition is experimentally measured.

\section{CONCLUDING REMARKS}

The aim of this note is to attract the attention to the possibility to have resonance enhancement of the axionphoton transformations in a medium. Such transitions 
between photons and axions in atoms can be represented via the effective electric and/or magnetic fields, similar to the interaction of the axion with physical electromagnetic fields. The crucial difference of these effective fields from the physical ones is that they couple to the axion through the independent coupling constant $g_{a e}$ which, for general axionlike particles, may be much larger than the axionphoton coupling $g_{a \gamma}$. This motivates to set up new axion production/detection experiments where the medium plays the role of physical magnetic field in the current axionsearch experiments. Such new experiments would allow one to measure the axion-electron coupling constant in the same way as the axion-photon coupling is studied. It is tempting to design such experiments and to estimate their efficiency.

We point out that the Eqs. (10) and (11) give an important hint towards the design of future atom- and molecule-based detectors with high sensitivity to axionlike particles: They should possess narrow spectral lines at relatively high atom density. In particular, it is natural to look for crystals with ultranarrow optical or microwave transitions. Examples of such crystals with the linewidth $\Gamma \sim 10^{-7} \mathrm{eV}$ in the optical region have recently been studied, see, e.g., [61]. In Sec. III C we demonstrated that the use of such crystals as a media for photon-axion transformation may give a significant enhancement of the effective field which may be much stronger than the physical fields employed at the ALPS experiments, see, e.g., [64] for a review. It is tempting to design such detectors and estimate their efficiency more accurately for applications in future ALPS experiments. We leave this issue for further work.

\section{ACKNOWLEDGMENTS}

The authors thank R. Ahlefeldt, V. Dzuba, C. Rizzo, M. Sellars, and Y. Stadnik for useful discussions. This work is supported by the Australian Research Council Grant No. DP150101405, the Gutenberg Fellowship, New Zealand Institute for Advanced Studies, the DFG via its Reinhardt Koselleck Program, and the European Research Council under the European Unions Horizon 2020 Research 41 and Innovative Program under Grant agreement No. 695405.

\section{APPENDIX: ESTIMATES OF EFFECTIVE FIELDS}

\section{Effective magnetic field produced by liquid xenon}

Let us consider the excited state $0^{-}$in $\mathrm{Xe}$ atom with energy $E_{0^{-}} \equiv \omega=9.447 \mathrm{eV}$. This state has zero total angular momentum, $J=0$, and odd parity in contrast with the even-parity ground state $0^{+}$. Therefore, the axion transition between these states is of M0 type. The corresponding matrix element for resonant axion absorption was calculated in our recent work [55]

$$
M^{a}=\left\langle 0^{-}\left|H_{\mathrm{M} 0}^{a e}\right| 0^{+}\right\rangle=i \frac{\sqrt{2}}{3} \omega^{2} R,
$$

where $R$ is the radial integral $R=\int_{0}^{\infty} f_{6 s_{1 / 2}}(r) \times$ $f_{5 p_{1 / 2}}(r) r^{3} d r$. Here $f_{6 s_{1 / 2}}(r)$ and $f_{5 p_{1 / 2}}(r)$ are radial parts of $6 s_{1 / 2}$ and $5 p_{1 / 2}$ wavefunctions, respectively (see, e.g., [60]). For Xe atom, the value of this integral may be deduced from [65], $R \approx-1.09$ a.u.

The photon transition $0^{+} \leftrightarrow 0^{-}$is highly forbidden. However, this transition may be opened by applying a magnetic field $B_{2}$ which provides the mixing of the excited states $\left|0^{-}\right\rangle$and $\left|1^{-}\right\rangle$,

$$
|i\rangle=\left|0^{-}\right\rangle-\frac{\left\langle 1^{-}\left|\boldsymbol{\mu} \cdot \mathbf{B}_{2}\right| 0^{-}\right\rangle}{E_{0^{-}}-E_{1^{-}}}\left|1^{-}\right\rangle,
$$

where $E_{1^{-}}=9.57 \mathrm{eV} ; \boldsymbol{\mu}=-\mu_{B}(\mathbf{J}+\mathbf{S})$ is the electron magnetic moment operator and $\mu_{B}$ is the Bohr magneton. Taking into account that the matrix element $\left\langle 0^{-}\left|\boldsymbol{\mu} \cdot \mathbf{B}_{2}\right| 1^{-}\right\rangle$ is $2 / 3 \mu_{B} B_{2}$, the photon $\mathrm{E} 1$ transition amplitude between the ground state $\left|0^{+}\right\rangle$and the excited state $|i\rangle$ is found to be

$M^{\gamma}=\frac{2}{3} \frac{e \mu_{B} B_{2}}{E_{0^{-}}-E_{1^{-}}}\left\langle 1^{-}|\boldsymbol{\epsilon} \cdot \mathbf{r}| 0^{+}\right\rangle=\frac{\sqrt{2}}{9} \frac{e \mu_{B} B_{2} R}{E_{0^{-}}-E_{1^{-}}}$,

where $\boldsymbol{\epsilon}$ is the photon polarization vector which is assumed to be along the $z$-axis, $\mathbf{\epsilon}=(0,0,1)$. Thus, for the product of transition amplitudes (A1) and (A2) we find

$$
M^{a} M^{\gamma}=\frac{2 i}{27} \frac{e \mu_{B} B_{2}}{E_{0^{-}}-E_{1^{-}}} \omega^{2} R^{2} .
$$

Note that the expression (A4) is imaginary. Hence, the real part of the effective Lagrangian (5) corresponds to the resonant absorption. Note also that for liquid $\mathrm{Xe}$ the dominant contribution to the width of the state $\Gamma$ comes from the collisional broadening, $\Gamma \approx \Gamma_{\text {col }}=2 v_{0} N_{\text {at }} \sigma_{\text {col }}$, where $v_{0}=\sqrt{2 k_{B} T / m_{\mathrm{at}}}$ is the most probable thermal speed of the atoms and $\sigma_{\mathrm{col}}$ is the collisional cross section. Here $k_{B}$ is the Boltzmann constant and $m_{\mathrm{at}}$ is the atomic mass. Assuming that the temperature of liquid $\mathrm{Xe}$ is $T=164 \mathrm{~K}$ and the atom density is $N_{\text {at }}=1.3 \times 10^{22} \mathrm{~cm}^{-3}$, the collisional width is estimated as $\Gamma_{\mathrm{col}} \approx 1.4 \times 10^{-3} \mathrm{eV}$. Taking into account Eq. (A4), we find the expression for the effective magnetic field

$$
B_{\text {eff }}=\frac{2}{27} N_{\text {at }} \frac{\mu_{B} B_{2}}{E_{0^{-}}-E_{1^{-}}} \frac{\omega^{2} R^{2}}{\Gamma_{\mathrm{col}}} .
$$

Assuming that the magnetic field $B_{2}$ is of order of the magnetic field applied at ALPS experiment [59], $B_{2}=5 \mathrm{~T}$, we estimate the effective magnetic field produced by the atomic transitions (A5): $B_{\mathrm{eff}} \approx 1.2 \times 10^{-2} \mathrm{~T}$. 


\section{Effective electric field produced by thallium vapor}

Consider the ground and excited states of the $\mathrm{Tl}$ atom, $A=\left|L=1, J=\frac{1}{2}\right\rangle$ and $B=\left|L=1, J=\frac{3}{2}\right\rangle$, with energy difference $\omega=0.966 \mathrm{eV}$. The transition between these states is of M1 type. The axion and photon absorption amplitudes for this transition are

$$
\begin{gathered}
M^{a}=-\frac{1}{2}\langle B|\mathbf{k} \cdot \boldsymbol{\sigma}| A\rangle, \\
M^{\gamma}=\frac{e}{2 m_{e}}(\mathbf{n} \times \mathbf{\epsilon})\langle B|\mathbf{J}+\mathbf{S}| A\rangle,
\end{gathered}
$$

where $\mathbf{n}$ is the photon or axion propagation unit vector and $\boldsymbol{\epsilon}$ is the photon polarization vector. For unpolarized atoms, in (11) we have to average over the states with different $z$-projection of the total angular momentum $M_{J}$. After this averaging, the contribution to the effective electric field vanishes, $\sum_{M_{J}} M^{a} M^{\gamma}=0$, see [55].

To get a non-vanishing result in (11) one can apply a magnetic field $B_{2}$, which splits the Zeeman sublevels. The laser frequency is in resonance with a transition from only one of these sublevels. In particular, we suppose that the constant magnetic field $B_{2}$ is applied along the $z$-axis, $\mathbf{B}_{2}=B_{2} \hat{\mathbf{z}}$, and the laser frequency corresponds to the energy difference of levels $A=\left|1, \frac{1}{2},-\frac{1}{2}\right\rangle$ and $B=\left|1, \frac{3}{2}, \frac{1}{2}\right\rangle$. We assume also that the photon propagates along the $y$-axis and is polarized in the $(x, z)$-plain, $\mathbf{n}=(0,1,0), \mathbf{\epsilon}=\left(\epsilon_{x}, 0, \epsilon_{z}\right)$. In this case, the axion and photon matrix elements (A6) and (A7) are

$$
\begin{aligned}
M^{a} & =\frac{i}{3 \sqrt{2}} \omega I, \\
M^{\gamma} & =\frac{e \epsilon_{z} I}{6 \sqrt{2} m_{e}},
\end{aligned}
$$

where $I$ is the overlap integral for $6 p_{1 / 2}$ and $6 p_{3 / 2}$ wave functions, $I=\int_{0}^{\infty} d r r^{2} f_{6 p_{1 / 2}}(r) f_{6 p_{3 / 2}}(r) \approx 0.98$.

As is shown in [60], for $\mathrm{Tl}$ vapor at a temperature of $T=1473 \mathrm{~K}$, the dominant contribution to the width of the line comes from the Doppler broadening, $\Gamma \approx \Gamma_{\text {Dop }}=$ $2 v_{0} \omega / \sqrt{\pi}$, where $v_{0}=\sqrt{2 k_{B} T / m_{\text {at }}}$ is the most probable thermal speed of the atoms. The numerical estimate for this width is $\Gamma_{\text {Dop }} \approx 1.3 \times 10^{-6} \mathrm{eV}$. Taking this into account, we have the following expression for the effective electric field

$$
E_{\mathrm{eff}}=N_{\mathrm{at}} \frac{e}{18 m_{e}} \frac{\omega I^{2} \epsilon_{z}}{\Gamma_{\mathrm{Dop}}},
$$

where the density of the vapor is $N_{\text {at }}=6.6 \times 10^{17} \mathrm{~cm}^{-3}$.

Assuming that the polarization of the photon is such that $\epsilon_{z}=1$, we estimate the effective electric field $E_{\text {eff }} \approx 1.5 \times 10^{5} \frac{\mathrm{v}}{\mathrm{cm}}$.
Note that the same result may be obtained using the optical pumping rather than applying the magnetic field $B_{2}$.

\section{Effective electric field in crystals}

Let us consider a $\mathrm{EuCl}_{3} \cdot 6 \mathrm{H}_{2} \mathrm{O}$ crystal, where there are narrow spectral lines in the optical region, and high optical density can be achieved, see [61]. The optical properties of this crystal can be understood through the electron-shell structure of the $\mathrm{Eu}^{3+}$ ion which has six valence electrons on the $4 \mathrm{f}$ orbital $[62,63]$. We are interested in the transition between the states $A={ }^{7} F_{0}$ and $B={ }^{5} D_{1}$ corresponding to the energy $\omega=2.36 \mathrm{eV}$. Since this transition is of M1 type, we apply the formula (11) to estimate the effective electric field $E_{\text {eff }}$ due to axion-photon transformation.

The relevant computation is similar to the one given in Sec. A 2 for $\mathrm{Tl}$ vapor. In particular, it is also necessary to apply the constant magnetic field $B_{2}$ along the $z$-axis to lift the degeneracy of the Zeeman sublevels of state $B={ }^{5} D_{1}$ with the total angular momentum $J=1$. To be specific, we assume that the laser frequency is in resonance with the transition between the states $|A\rangle=\left|{ }^{7} F_{0} ; J=0, M=0\right\rangle$ and $|B\rangle=\left|{ }^{5} D_{1} ; J=1, M=1\right\rangle$. For simplicity, we also assume that the laser beam is aligned along the $y$-direction and is polarized in the $z$-direction, so that $\mathbf{n}=(0,1,0)$ and $\mathbf{\epsilon}=(0,0,1)$. In this case, using the Eqs. (A6) and (A7) we find

$$
\begin{gathered}
M^{a}=-\frac{i}{\sqrt{6}} \omega\left\langle{ }^{5} D_{1} \| S \mid{ }^{7} F_{0}\right\rangle, \\
M^{\gamma}=-\frac{e}{2 \sqrt{6} m_{e}}\left\langle{ }^{5} D_{1} \| J+S \mid{ }^{7} F_{0}\right\rangle,
\end{gathered}
$$

where $\left\langle{ }^{5} D_{1} \| S \mid{ }^{7} F_{0}\right\rangle$ is the reduced matrix element of the spin operator $\mathbf{S}$ and $\left\langle{ }^{5} D_{1} \| J+S||^{7} F_{0}\right\rangle=\left\langle{ }^{5} D_{1} \| S \mid{ }^{7} F_{0}\right\rangle$. This matrix element may be computed using the spectral data for the $\mathrm{Eu}^{3+}$ ion $[62,63]:\left\langle{ }^{5} D_{1} \| S||{ }^{7} F_{0}\right\rangle \approx 0.17$.

To apply the Eq. (11) we need the atom density $N_{\text {at }}$ and the width of the state $\Gamma$. The former may be easily estimated for the crystal $\mathrm{EuCl}_{3} \cdot 6 \mathrm{H}_{2} \mathrm{O}$ described in [61]: $N_{\mathrm{at}} \approx 2.7 \times 10^{21} \mathrm{~cm}^{-3}$. The authors of this paper claim that the width of the optical transition ${ }^{7} F_{0} \rightarrow{ }^{5} D_{0}$ in this crystal is about $25 \mathrm{MHz}$. For our estimates, we can assume that the transition ${ }^{7} F_{0} \rightarrow{ }^{5} D_{1}$ possesses a comparable width $\Gamma \sim 10^{-7} \mathrm{eV}$. For these values of the parameters, we find the effective electric field in the crystal due to the photonaxion transformation,

$E_{\text {eff }}=\frac{N_{\mathrm{at}} e \omega}{12 m_{e} \Gamma}\left|\left\langle{ }^{5} D_{1}|| S \mid{ }^{7} F_{0}\right\rangle\right|^{2} \approx 1.7 \times 10^{9} \frac{\mathrm{V}}{\mathrm{cm}}$.

We point out that these estimates are very rough; a more accurate estimate requires experimental measurements of the width of the line for the transition ${ }^{7} F_{0} \rightarrow{ }^{5} D_{1}$ in the $\mathrm{EuCl}_{3} \cdot 6 \mathrm{H}_{2} \mathrm{O}$ crystal. 
[1] R. D. Peccei and H. R. Quinn, Phys. Rev. Lett. 38, 1440 (1977).

[2] R. D. Peccei, Axions: Theory, Cosmology, and Experimental Searches (Springer, New York, 2008), pp. 3-17.

[3] S. Weinberg, Phys. Rev. Lett. 40, 223 (1978).

[4] F. Wilczek, Phys. Rev. Lett. 40, 279 (1978).

[5] J. E. Kim, Phys. Rev. Lett. 43, 103 (1979).

[6] M. A. Shifman, A. I. Vainshtein, and V. I. Zakharov, Nucl. Phys. B166, 493 (1980).

[7] M. Dine, W. Fischler, and M. Srednicki, Phys. Lett. 104B, 199 (1981).

[8] A. R. Zhitnitsky, Yad. Fiz. 31, 497 (1980) [Sov. J. Nucl. Phys. 31, 260 (1980)].

[9] M. Srednicki, Nucl. Phys. B260, 689 (1985).

[10] P. Sikivie, Phys. Rev. Lett. 51, 1415 (1983); 52, 695(E) (1984).

[11] P. Sikivie, Phys. Rev. D 32, 2988 (1985); 36, 974(E) (1987).

[12] S. Moriyama, M. Minowa, T. Namba, Y. Inoue, Y. Takasu, and A. Yamamoto, Phys. Lett. B 434, 147 (1998).

[13] Y. Inoue, T. Namba, S. Moriyama, M. Minowa, Y. Takasu, T. Horiuchi, and A. Yamamoto, Phys. Lett. B 536, 18 (2002).

[14] Y. Inoue, Y. Akimoto, R. Ohta, T. Mizumoto, A. Yamamoto, and M. Minowa, Phys. Lett. B 668, 93 (2008).

[15] R. Ohta et al., Nucl. Instrum. Methods Sect. A 670, 73 (2012).

[16] T. Mizumoto, R. Ohta, T. Horie, J. Suzuki, Y. Inoue, and M. Minowa, J. Cosmol. Astropart. Phys. 07 (2013) 013.

[17] K. Zioutas et al., Nucl. Instrum. Methods Sect. A 425, 480 (1999).

[18] E. Arik et al. (CAST Collaboration), J. Cosmol. Astropart. Phys. 02 (2009) 008.

[19] V. Anastassopoulos et al. (CAST Collaboration), Nat. Phys. 13, 584 (2017).

[20] F. T. Avignone, III et al. (SOLAX Collaboration), Phys. Rev. Lett. 81, 5068 (1998).

[21] A. O. Gattone et al., Nucl. Phys. B, Proc. Suppl. 70, 59 (1999).

[22] A. Morales et al. (COSME Collaboration), Astropart. Phys. 16, 325 (2002).

[23] R. Bernabei et al., Phys. Lett. B 515, 6 (2001).

[24] R. Bernabei et al., Int. J. Mod. Phys. A 21, 1445 (2006).

[25] R. Bernabei et al. (DAMA and LIBRA Collaborations), Eur. Phys. J. C 67, 39 (2010).

[26] Z. Ahmed et al. (CDMS Collaboration), Phys. Rev. Lett. 103, 141802 (2009).

[27] Z. Ahmed et al. (CDMS-II Collaboration), Phys. Rev. Lett. 106, 131302 (2011).

[28] R. Agnese et al. (CDMS Collaboration), Phys. Rev. Lett. 111, 251301 (2013).

[29] R. Agnese et al. (SuperCDMS Collaboration), Phys. Rev. Lett. 112, 241302 (2014).

[30] I. G. Irastorza et al., J. Cosmol. Astropart. Phys. 06 (2011) 013.

[31] E. Armengaud et al., J. Instrum. 9, T05002 (2014).

[32] S. J. Asztalos et al. (ADMX Collaboration), Phys. Rev. Lett. 104, 041301 (2010).

[33] A. Wagner et al. (ADMX Collaboration), Phys. Rev. Lett. 105, 171801 (2010).
[34] J. Hoskins et al., Phys. Rev. D 84, 121302 (2011).

[35] S. Al Kenany et al., Nucl. Instrum. Methods Sect. A 854, 11 (2017).

[36] B. M. Brubaker et al., Phys. Rev. Lett. 118, 061302 (2017).

[37] B. T. McAllister, G. Flower, E. N. Ivanov, M. Goryachev, J. Bourhill, and M.E. Tobar, Phys. Dark Universe 18, 67 (2017).

[38] K. Ehret, M. Frede, E.-A. Knabbe, D. Kracht, A. Lindner, N. Meyer, D. Notz, A. Ringwald, and G. Wiedemann, arXiv:hep-ex/0702023.

[39] K. Ehret et al. (ALPS Collaboration), Nucl. Instrum. Methods Sect. A 612, 83 (2009).

[40] K. Ehret et al., Phys. Lett. B 689, 149 (2010).

[41] P. Pugnat et al. (OSQAR Collaboration), Phys. Rev. D 78, 092003 (2008).

[42] A. S. Chou, W. Wester, A. Baumbaugh, H. R. Gustafson, Y. Irizarry-Valle, P. O. Mazur, J. H. Steffen, R. Tomlin, X. Yang, and J. Yoo (GammeV (T-969) Collaboration), Phys. Rev. Lett. 100, 080402 (2008).

[43] G. Cantatore, F. Della Valle, E. Milotti, L. Dabrowski, and C. Rizzo, Phys. Lett. B 265, 418 (1991).

[44] E. Zavattini et al. (PVLAS Collaboration), Phys. Rev. D 77, 032006 (2008).

[45] F. Della Valle, A. Ejlli, U. Gastaldi, G. Messineo, E. Milotti, R. Pengo, G. Ruoso, and G. Zavattini, Eur. Phys. J. C 76, 24 (2016).

[46] S. J. Chen, H. H. Mei, and W. T. Ni, Mod. Phys. Lett. A 22, 2815 (2007).

[47] R. Battesti et al., Eur. Phys. J. D 46, 323 (2008).

[48] F. Bielsa et al., arXiv:0911.4567.

[49] E. Iacopini and E. Zavattini, Phys. Lett. 85B, 151 (1979).

[50] K. Zioutas and Y. Semertzidis, Phys. Lett. 130A, 94 (1988).

[51] P. Sikivie, Phys. Rev. Lett. 113, 201301 (2014).

[52] M. Pospelov, A. Ritz, and M. B. Voloshin, Phys. Rev. D 78, 115012 (2008).

[53] L. Santamaria, C. Braggio, G. Carugno, V. Di Sarno, P. Maddaloni, and G. Ruoso, New J. Phys. 17, 113025 (2015).

[54] A. Arvanitaki, S. Dimopoulos, and K. Van Tilburg, Phys. Rev. X 8, 041001 (2018).

[55] H. B. Tran Tan, V. V. Flambaum, I. B. Samsonov, Y. V. Stadnik, and D. Budker, arXiv:1803.09388.

[56] K. van Bibber, N. R. Dagdeviren, S. E. Koonin, A. Kerman, and H. N. Nelson, Phys. Rev. Lett. 59, 759 (1987).

[57] L. Landau and E. Lifshitz, Quantum Mechanics (Pergammon Press, Hungary, 1969).

[58] K. van Bibber, P. M. McIntyre, D. E. Morris, and G. G. Raffelt, Phys. Rev. D 39, 2089 (1989).

[59] K. Ehret et al., Phys. Lett. B 689, 149 (2010).

[60] I. B. Khriplovich, Parity Nonconservation in Atomic Phenomena (Gordon and Breach, Amsterdam, 1991).

[61] R. L. Ahlefeldt, M. R. Hush, and M. J. Sellars, Phys. Rev. Lett. 117, 250504 (2016).

[62] K. Binnemans, Coord. Chem. Rev. 295, 1 (2015).

[63] G. S. Ofelt, J. Chem. Phys. 38, 2171 (1963).

[64] R. Battesti et al., arXiv:1803.07547.

[65] A. Kramida, Yu. Ralchenko, J. Reader (NIST ASD Team), NIST atomic spectra database (ver. 5.5.3), National Institute of Standards and Technology, 2018,https://physics.nist.gov/ asd. 